\title{
Complete genome sequence of Bacteroides helcogenes type strain (P 36-108 ${ }^{\mathrm{T}}$ )
}

\author{
Amrita Pati ${ }^{1}$, Sabine Gronow ${ }^{2}$, Ahmet Zeytun ${ }^{1,3}$, Alla Lapidus ${ }^{1}$, Matt Nolan ${ }^{1}$, Nancy \\ Hammon ${ }^{1}$, Shweta Deshpande ${ }^{1}$, Jan-Fang Cheng ${ }^{1}$, Roxane Tapia ${ }^{1,3}$, Cliff Han ${ }^{1,3}$, Lynne \\ Goodwin $^{1,3}$, Sam Pitluck ${ }^{1}$, Konstantinos Liolios ${ }^{1}$, Ioanna Pagani ${ }^{1}$, Natalia Ivanova ${ }^{1}$, \\ Konstantinos Mavromatis ${ }^{1}$, Amy Chen ${ }^{4}$, Krishna Palaniappan ${ }^{4}$, Miriam Land ${ }^{1,5}$, Loren \\ Hauser $^{1,5}$, Yun-Juan Chang ${ }^{1,5}$, Cynthia D. Jeffries ${ }^{1,5}$, John C. Detter ${ }^{1}$, Evelyne Brambilla ${ }^{2}$, \\ Manfred Rohde ${ }^{6}$, Markus Göker ${ }^{2}$, Tanja Woyke ${ }^{1}$, James Bristow ${ }^{1}$, Jonathan A. Eisen ${ }^{1,7}$, Victor \\ Markowitz $^{4}$, Philip Hugenholtz ${ }^{1,8}$, Nikos C. Kyrpides ${ }^{1}$, Hans-Peter Klenk ${ }^{2 *}$, and Susan Lucas ${ }^{1}$ \\ ${ }^{1}$ DOE Joint Genome Institute, Walnut Creek, California, USA \\ ${ }^{2}$ DSMZ - German Collection of Microorganisms and Cell Cultures $\mathrm{GmbH}$, Braunschweig, \\ Germany \\ ${ }^{3}$ Los Alamos National Laboratory, Bioscience Division, Los Alamos, New Mexico, USA \\ ${ }^{4}$ Biological Data Management and Technology Center, Lawrence Berkeley National \\ Laboratory, Berkeley, California, USA \\ ${ }^{5}$ Oak Ridge National Laboratory, Oak Ridge, Tennessee, USA \\ ${ }^{6} \mathrm{HZI}$ - Helmholtz Centre for Infection Research, Braunschweig, Germany \\ ${ }^{7}$ University of California Davis Genome Center, Davis, California, USA \\ ${ }^{8}$ Australian Centre for Ecogenomics, School of Chemistry and Molecular Biosciences, The \\ University of Queensland, Brisbane, Australia \\ ${ }^{*}$ Corresponding author: Hans-Peter Klenk
}

Keywords: strictly anaerobic, mesophilic, nonmotile, Gram-negative, chemoorganotrophic, pig abscess, animal pathogen, Bacteroidaceae, GEBA

Bacteroides helcogenes Benno et al. 1983 is of interest because of its isolated phylogenetic location and, although it has been found in pig feces and is known to be pathogenic for pigs, occurrence of this bacterium is rare and it does not cause significant damage in intensive animal husbandry. The genome of $B$. helcogenes $\mathrm{P} 36-108^{\top}$ is already the fifth completed and published type strain genome from the genus Bacteroides in the family Bacteroidaceae. The 3,998,906 bp long genome with its 3,353 protein-coding and 83 RNA genes consists of one circular chromosome and is a part of the Genomic Encyclopedia of Bacteria and Archaea project.

\section{Introduction}

Strain P 36-108 (= DSM 20613 = ATCC $35417=$ JCM 6297) is the type strain of Bacteroides helcogenes, one of currently 39 species in the genus Bacteroides [1,2]. The species epithet of B. helcogenes is derived from the Greek noun helkos meaning 'abscess' and the Greek verb gennaio meaning 'produce', referring to the pathogenic, probably intestinal, abscess-producing properties of the species [2]. B. helcogenes strain P36-108 was isolated from a pig abscess in Japan, and described by Benno et al. in 1983 [2]. Nine further isolates of $B$. helcogenes have been obtained from pig abscesses whereas two other isolates origi- nated from pig feces. Here we present a summary classification and a set of features for $B$. helcogenes $\mathrm{P} 36-108^{\mathrm{T}}$, together with the description of the complete genomic sequencing and annotation.

\section{Classification and features}

A representative genomic $16 \mathrm{~S}$ rRNA sequence of $B$. helcogenes was compared using NCBI BLAST under default values (e.g., considering only the high-scoring segment pairs (HSPs) from the best 250 hits) with the most recent release of the Greengenes database [3] and the relative frequen- 
cies, weighted by BLAST scores, of taxa and keywords (reduced to their stem [4]) were determined. The single most frequent genus was Bacteroides (100\%) (33 hits in total). Regarding the 21 hits to sequences from other members of the genus, the average identity within HSPs was $92.7 \%$, whereas the average coverage by HSPs was 84.5\%. Among all other species, the one yielding the highest score was Bacteroides ovatus, which corresponded to an identity of $93.4 \%$ and a HSP coverage of $86.6 \%$. The highest-scoring environmental sequence was AM275453 ('fecal microbiota irritable bowel syndrome patients differs significantly from that of healthy subjects'), which showed an identity of $95.5 \%$ and a HSP coverage of $84.3 \%$. The most frequently occurring keywords within the labels of environmental samples which yielded hits were 'human' (11.0\%), 'fecal' (9.5\%), 'microbiota' (8.8\%), 'sequenc' (5.4\%) and 'gut' (5.4\%) (217 hits in total). The most frequently occurring keywords within the labels of environmental samples which yielded hits of a higher score than the highest scoring species were 'fecal/human' (13.3\%), 'feedlot' (5.2\%), 'bowel, faecal, healthi, irrit, microbiota, patient, significantli, subject, syndrom' (2.7\%) and 'beef, cattl, coli, escherichia, feedbunk, habitat, marc, materi, neg, pen, primari, secondari, stec, surfac, synecolog, top, west' (2.6\%) (6 hits in total). Most of these keywords are in accordance with the isolation sites of the different isolates and strongly suggest that $B$. helcogenes, like many other species of the genus Bacteroides, is associated with the intestinal tract of the host in the case of $B$. helcogenes, this host is the pig [2].

Figure 1 shows the phylogenetic neighborhood of B. helcogenes $\mathrm{P} 36-108^{\mathrm{T}}$ in a $16 \mathrm{~S}$ rRNA based tree. The sequences of the five 16S rRNA gene copies in the genome differ from each other by up to 20 nucleotides, and differ by up to 13 nucleotides from the previously published $16 \mathrm{~S}$ rRNA sequence (AB200227).

The cells of $B$. helcogenes generally have the shape of short rods (0.5-0.6 $\mu \mathrm{m} \times 0.8-4.0 \mu \mathrm{m})$ which occur singly or in pairs (Figure 2). B. helcogenes is a Gram-negative, non-pigmented and non sporeforming bacterium (Table 1). The organism is originally described as nonmotile and only five genes associated with motility have been found in the genome (see below). The organism grows well at $37^{\circ} \mathrm{C}$ but does not grow at $4^{\circ} \mathrm{C}$ or at $45^{\circ} \mathrm{C}$ [2]. $B$. helcogenes is strictly anaerobic, chemoorganotrophic and is able to ferment glucose, mannose, fructose, galactose, sucrose, maltose, cellobiose, lactose, xylose, melibiose, raffinose, starch, glycogen, salicin, amygdalin, and xylan [2]. The organism hydrolyzes esculin and starch but does not digest casein, liquify gelatin, reduce nitrate nor produce indole from tryptophan [2]. B. helcogenes does not utilize arabinose, ramnose, ribose, trehalose, inulin, glycerol, mannitol, sorbitol, inositol, adonitol, erythritol or gum Arabic [2]. It does not require hemin for growth but does require the presence of $\mathrm{CO}_{2}$; it does not show hemolysis. Growth is not enhanced by the addition of $20 \%$ bile [2]. Major fermentation products from PYFG broth (peptone yeast extract Fildes glucose broth [26]) are acetic acid and succinic acid; propionic and isobutyric acid are produced in small amounts [2]. B. helcogenes is phosphatase, DNase, $\beta$ glucuronidase, and glutamic acid decarboxylase active and urease, catalase, lecithinase and lipase inactive [2]. The organism produces ammonium and chondroitin sulfatase [2]. B. helcogenes can grow in the presence of kanamycin $(1 \mathrm{mg} / \mathrm{ml})$, vancomycin $(10 \mu \mathrm{g} / \mathrm{ml})$, colistin $(10 \mu \mathrm{g} / \mathrm{ml})$, erythromycin $(60 \mu \mathrm{g} / \mathrm{ml})$ or polymyxin B $(10 \mu \mathrm{g} / \mathrm{ml})$ but not in the presence of cepharothin $(10 \mu \mathrm{g} / \mathrm{ml})$ or Brilliant green $(0.001 \%)$ [2].

\section{Chemotaxonomy}

Little chemotaxonomic information is available for strain P 36-108 ${ }^{\mathrm{T}}$. Thus far, only the fatty acid composition has been elucidated. The major fatty acids found $(>10 \%)$ were anteiso- $\mathrm{C}_{15: 0}, \mathrm{C}_{15: 0}$ and iso- $\mathrm{C}_{15: 0.3-0 \mathrm{H}}$. Also, iso- $\mathrm{C}_{15: 0}, \mathrm{C}_{16: 0}$, and cis $\mathrm{C}_{18: 1}$ were detected in a proportion ranging between $5 \%$ to $10 \%$ of the total fatty acids (unpublished data).

\section{Genome sequencing and annotation Genome project history}

This organism was selected for sequencing on the basis of its phylogenetic position [27], and is part of the Genomic Encyclopedia of Bacteria and Archaea project [28]. The genome project is deposited in the Genomes OnLine Database [10] and the complete genome sequence is deposited in GenBank. Sequencing, finishing and annotation were performed by the DOE Joint Genome Institute (JGI). A summary of the project information is shown in Table 2 . 


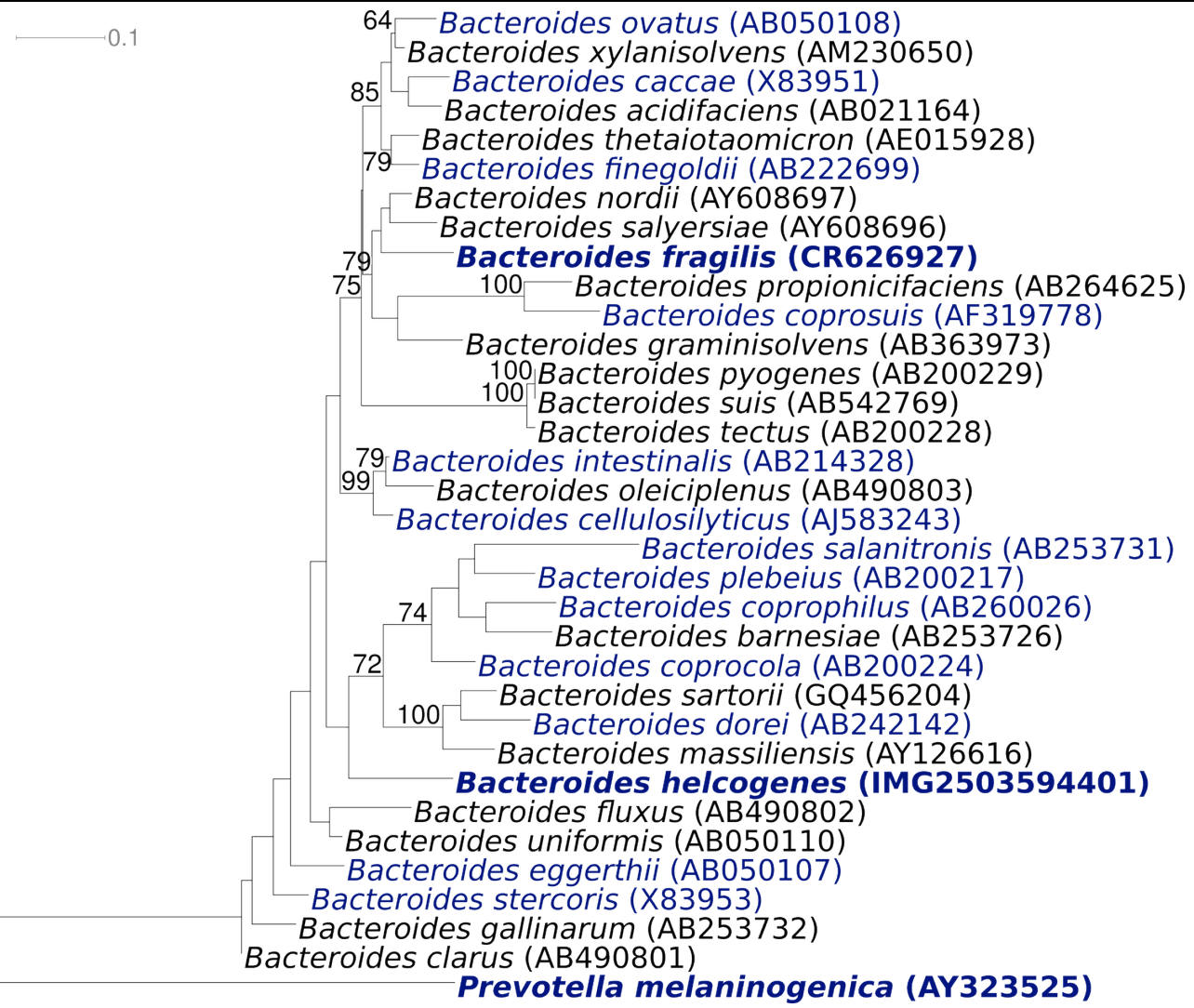

Figure 1. Phylogenetic tree highlighting the position of $B$. helcogenes relative to those type strains within the genus that appeared within a monophyletic Bacteroides main clade in preliminary analyses. Note that several of the Bacteroides type strain 16S rRNA sequences (from B. cellulosolvens, B. galacturonicus, B. pectinophilus, B. vulgatus) did not cluster together with this clade (data not shown, but see [5]) and were omitted from the main phylogenetic inference analysis. The same holds for the sequence from Anaerorhabdus furcosa (GU585668; also Bacteroidaceae). Other Bacteroides species lacked a sufficiently long $16 \mathrm{~S}$ rRNA sequence and also had to be omitted (B. coagulans, B. xylanolyticus). The tree was inferred from 1,414 aligned characters [6,7] of the 16S rRNA gene sequence under the maximum likelihood criterion [8] and rooted with the type strain of the family 'Prevotellaceae'. The branches are scaled in terms of the expected number of substitutions per site. Numbers above branches are support values from 1,000 bootstrap replicates [9] if larger than $60 \%$. Lineages with type strain genome sequencing projects registered in GOLD [10] are shown in blue, published genomes [11] and Prevotella melaninogenica released Genbank accession CP002122 in bold.

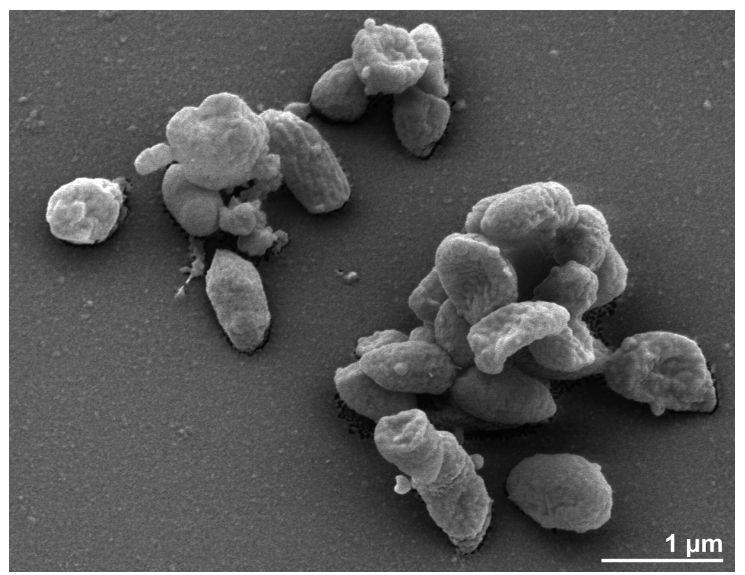

Figure 2. Scanning electron micrograph of $B$. helcogenes $\mathrm{P} 36-108^{\top}$ 
Table 1. Features of $B$. helcogenes $\mathrm{P} 36-108^{\top}$ according to the MIGS recommendations [12].

\begin{tabular}{|c|c|c|c|}
\hline MIGS ID & Property & Term & Evidence code \\
\hline & & Domain Bacteria & TAS [13] \\
\hline & & Phylum Bacteroidetes & TAS [14] \\
\hline & & Class 'Bacteroidia' & TAS [15] \\
\hline & & Order 'Bacteroidales' & TAS [16] \\
\hline & & Family Bacteroidaceae & TAS $[17,18]$ \\
\hline & & Genus Bacteroides & TAS $[17,19-22]$ \\
\hline & & Species Bacteroides helcogenes & TAS $[2,23]$ \\
\hline & Current classification & Type strain P 36-108 & TAS [2] \\
\hline & Gram stain & negative & TAS [2] \\
\hline & Cell shape & rod-shaped, single or in pairs & TAS [2] \\
\hline & Motility & non-motile & TAS [2] \\
\hline & Sporulation & none & TAS [2] \\
\hline & Temperature range & mesophile & TAS [2] \\
\hline & Optimum temperature & $37^{\circ} \mathrm{C}$ & TAS [2] \\
\hline & Salinity & normal & TAS [2] \\
\hline \multirow[t]{3}{*}{ MIGS-22 } & Oxygen requirement & strictly anaerobic & TAS [2] \\
\hline & Carbon source & carbohydrates & TAS [2] \\
\hline & Energy source & chemoorganotroph & TAS [2] \\
\hline MIGS-6 & Habitat & host & TAS [2] \\
\hline MIGS-15 & Biotic relationship & free-living & TAS [2] \\
\hline \multirow[t]{3}{*}{ MIGS-14 } & Pathogenicity & animal pathogen & TAS [2] \\
\hline & Biosafety level & 2 & TAS [24] \\
\hline & Isolation & Sus scrofa abscess & TAS [2] \\
\hline MIGS-4 & Geographic location & Japan & TAS [2] \\
\hline MIGS-5 & Sample collection time & 1974 & TAS [2] \\
\hline MIGS-4.1 & Latitude & not reported & NAS \\
\hline MIGS-4.2 & Longitude & not reported & NAS \\
\hline MIGS-4.3 & Depth & not reported & NAS \\
\hline MIGS-4.4 & Altitude & not reported & NAS \\
\hline
\end{tabular}

Evidence codes - IDA: Inferred from Direct Assay (first time in publication); TAS: Traceable Author Statement (i.e., a direct report exists in the literature); NAS: Non-traceable Author Statement (i.e., not directly observed for the living, isolated sample, but based on a generally accepted property for the species, or anecdotal evidence). These evidence codes are from of the Gene Ontology project [25]. If the evidence code is IDA, then the property was directly observed by one of the authors or an expert mentioned in the acknowledgements.

\section{Growth conditions and DNA isolation}

B. helcogenes P 36-108T, DSM 20613, was grown anaerobically in medium 104 (PYG Medium) [29] at $37^{\circ} \mathrm{C}$. DNA was isolated from $0.5-1 \mathrm{~g}$ of cell paste using MasterPure Gram-positive DNA purification kit (Epicentre MGP04100) following the standard protocol as recommended by the manufacturer, with modification st/DL for cell lysis as described in $\mathrm{Wu}$ et al. [28]. DNA is available through the DNA Bank Network [30,31].

\section{Genome sequencing and assembly}

The genome was sequenced using a combination of Illumina and 454 sequencing platforms. All general aspects of library construction and sequencing can be found at the JGI website [32]. Pyrosequencing reads were assembled using the Newbler assembler version 2.3-PreRelease-10-212009-gcc-4.1.2-threads (Roche). The initial Newbler assembly consisting of 48 contigs in two scaf- 
folds was converted into a phrap assembly by [33] making fake reads from the consensus, to collect the read pairs in the 454 paired end library. Illumina GAii sequencing data $(225.3 \mathrm{Mb})$ was assembled with Velvet [34] and the consensus sequences were shredded into $1.5 \mathrm{~kb}$ overlapped fake reads and assembled together with the 454 data. The 454 draft assembly was based on 146.7 Mb 454 draft data and all of the 454 paired end data. Newbler parameters are -consed -a 50 -1 350 -g -m -ml 20. The Phred/Phrap/Consed software package [33] was used for sequence assembly and quality assessment in the subsequent finishing process. After the shotgun stage, reads were assembled with parallel phrap (High Performance Software, LLC). Possible mis-assemblies were corrected with gapResolution [32], Dupfinisher [35], or sequencing cloned bridging PCR fragments with subcloning or transposon bombing (Epicentre Biotechnologies, Madison, WI). Gaps between contigs were closed by editing in Consed, by PCR and by Bubble PCR primer walks (J.-F.Chang, unpublished). A total of 160 additional reactions and 4 shatter libraries were necessary to close gaps and to raise the quality of the finished sequence. Illumina reads were also used to correct potential base errors and increase consensus quality using a software Polisher developed at JGI [36]. The error rate of the completed genome sequence is less than 1 in 100,000. Together, the combination of the Illumina and 454 sequencing platforms provided $93 \times$ coverage of the genome. The final assembly contained 500,148 pyrosequence and $6,257,254$ Illumina reads.

Table 2. Genome sequencing project information

\begin{tabular}{lll}
\hline MIGS ID & Property & Term \\
\hline MIGS-31 & Finishing quality & Finished \\
MIGS-28 & Libraries used & Three genomic libraries: one 454 pyrosequence standard li- \\
brary, one 454 PE library (9 kb insert size), one Illumina library \\
MIGS-29 & Sequencing platforms & Illumina GAii, 454 GS FLX Titanium \\
MIGS-31.2 & Sequencing coverage & $56.3 \times$ Illumina; 36.7 × pyrosequence \\
MIGS-30 & Assemblers & Newbler version 2.3-PreRelease-10-21-2009-gcc-4.1.2- \\
& threads, Velvet, phrap \\
MIGS-32 & Gene calling method & Prodigal 1.4, GenePRIMP \\
& INSDC ID & CP002352 \\
& Genbank Date of Release & January 18, 2011 \\
& GOLD ID & Gc01593 \\
& NCBI project ID & 41913 \\
& Database: IMG-GEBA & 2503538016 \\
& Source material identifier & DSM 20613 \\
& Project relevance & Tree of Life, GEBA \\
\hline
\end{tabular}

\section{Genome annotation}

Genes were identified using Prodigal [37] as part of the Oak Ridge National Laboratory genome annotation pipeline, followed by a round of manual curation using the JGI GenePRIMP pipeline [38]. The predicted CDSs were translated and used to search the National Center for Biotechnology Information (NCBI) nonredundant database, UniProt, TIGR-Fam, Pfam, PRIAM, KEGG, COG, and InterPro databases. Additional gene prediction analysis and functional annotation was performed within the Integrated Microbial Genomes - Expert Review (IMG-ER) platform [39].

\section{Genome properties}

The genome consists of a 3,998,906 bp long chromosome with a GC content of $44.7 \%$ (Figure 3 and Table 3). Of the 3,436 genes predicted, 3,353 were protein-coding genes, and 83 RNAs; 109 pseudogenes were also identified. The majority of the protein-coding genes (64.5\%) were assigned with a putative function while the remaining ones were annotated as hypothetical proteins. The distribution of genes into COGs functional categories is presented in Table 4. 


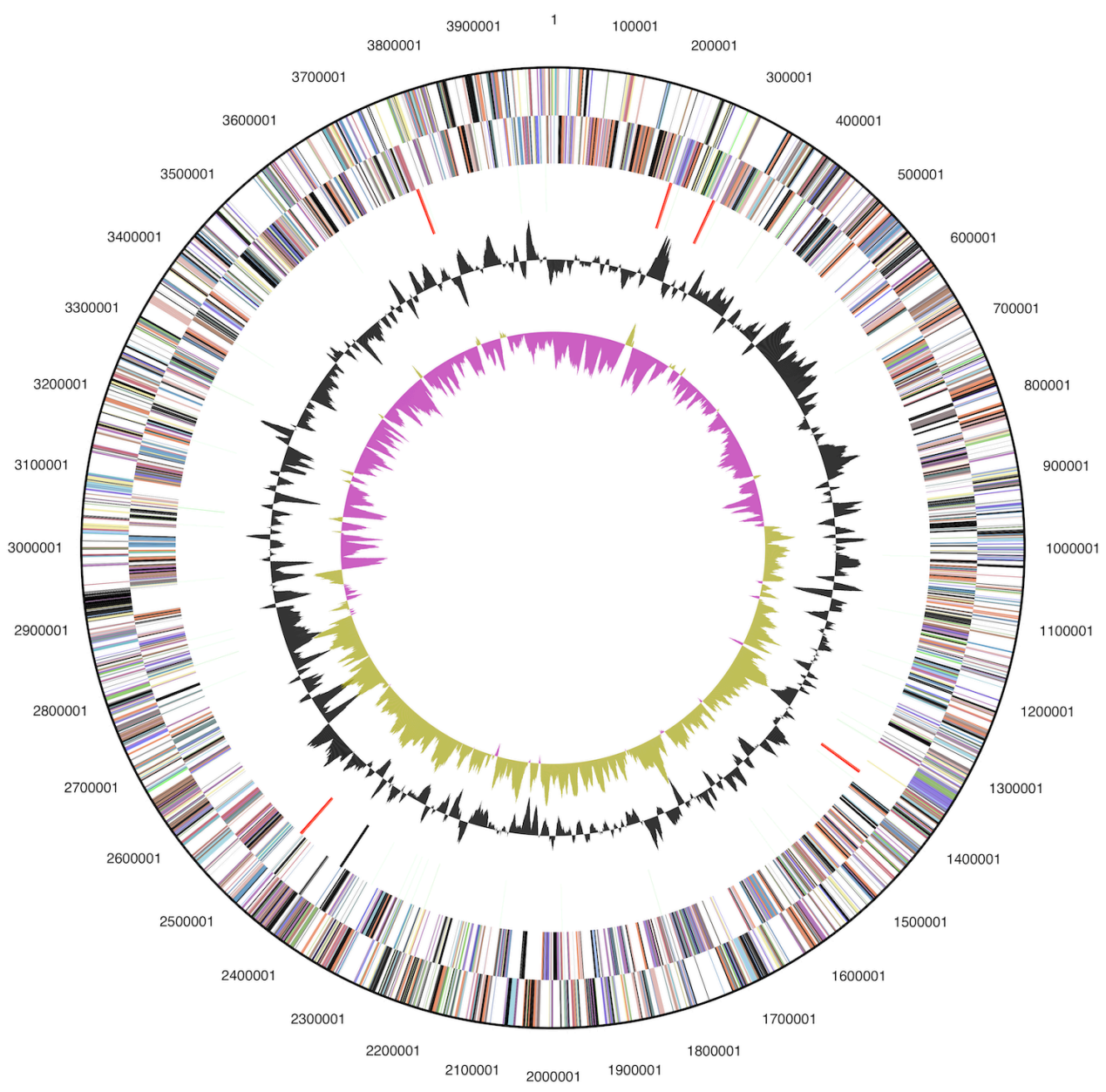

Figure 3. Graphical circular map of the chromosome. From outside to the center: Genes on forward strand (color by COG categories), Genes on reverse strand (color by COG categories), RNA genes (tRNAs green, rRNAs red, other RNAs black), GC content, GC skew.

Table 3. Genome Statistics

\begin{tabular}{lrr}
\hline Attribute & Value & \% of Total \\
\hline Genome size (bp) & $3,998,906$ & $100.00 \%$ \\
DNA coding region (bp) & $3,583,947$ & $89.62 \%$ \\
DNA G+C content (bp) & $1,788,209$ & $44.72 \%$ \\
Number of replicons & 1 & \\
Extrachromosomal elements & 0 & \\
Total genes & 3,436 & $100.00 \%$ \\
RNA genes & 83 & $2.42 \%$ \\
rRNA operons & 5 & \\
Protein-coding genes & 3,353 & $97.58 \%$ \\
Pseudo genes & 109 & $3.17 \%$ \\
Genes with function prediction & 2,215 & $64.46 \%$ \\
Genes in paralog clusters & 454 & $13.21 \%$ \\
Genes assigned to COGs & 2103 & $61.20 \%$ \\
Genes assigned Pfam domains & 2360 & $68.68 \%$ \\
Genes with signal peptides & 980 & $28.52 \%$ \\
Genes with transmembrane helices & 798 & $23.22 \%$ \\
CRISPR repeats & 1 & \\
\hline
\end{tabular}


Table 4. Number of genes associated with the general COG functional categories

\begin{tabular}{lrrl}
\hline Code & value & \%age & Description \\
\hline J & 147 & 6.5 & Translation, ribosomal structure and biogenesis \\
$\mathrm{A}$ & 0 & 0 & RNA processing and modification \\
$\mathrm{K}$ & 157 & 6.9 & Transcription \\
$\mathrm{L}$ & 125 & 5.5 & Replication, recombination and repair \\
$\mathrm{B}$ & 0 & 0 & Chromatin structure and dynamics \\
$\mathrm{D}$ & 20 & 0.9 & Cell cycle control, cell division, chromosome partitioning \\
$\mathrm{Y}$ & 0 & 0 & Nuclear structure \\
$\mathrm{V}$ & 67 & 2.9 & Defense mechanisms \\
$\mathrm{T}$ & 125 & 5.5 & Signal transduction mechanisms \\
$\mathrm{M}$ & 245 & 10.8 & Cell wall/membrane/envelope biogenesis \\
$\mathrm{N}$ & 5 & 0.2 & Cell motility \\
$\mathrm{Z}$ & 0 & 0 & Cytoskeleton \\
$\mathrm{W}$ & 0 & 0 & Extracellular structures \\
$\mathrm{U}$ & 48 & 2.1 & Intracellular trafficking, secretion, and vesicular transport \\
$\mathrm{O}$ & 66 & 2.9 & Posttranslational modification, protein turnover, chaperones \\
$\mathrm{C}$ & 120 & 5.3 & Energy production and conversion \\
$\mathrm{G}$ & 185 & 8.1 & Carbohydrate transport and metabolism \\
$\mathrm{E}$ & 149 & 6.5 & Amino acid transport and metabolism \\
$\mathrm{F}$ & 67 & 2.9 & Nucleotide transport and metabolism \\
$\mathrm{H}$ & 120 & 5.3 & Coenzyme transport and metabolism \\
$\mathrm{I}$ & 64 & 2.8 & Lipid transport and metabolism \\
$\mathrm{P}$ & 161 & 7.6 & Inorganic ion transport and metabolism \\
$\mathrm{Q}$ & 20 & 0.9 & Secondary metabolites biosynthesis, transport and catabolism \\
$\mathrm{R}$ & 266 & 11.7 & General function prediction only \\
$\mathrm{S}$ & 122 & 5.4 & Function unknown \\
- & 1,333 & 38.8 & Not in COGs \\
\hline
\end{tabular}

\section{Acknowledgements}

We would like to gratefully acknowledge the help of Sabine Welnitz (DSMZ) for growing B. helcogenes cultures. This work was performed under the auspices of the US Department of Energy Office of Science, Biological and Environmental Research Program, and by the University of California, Lawrence Berkeley National Laboratory under contract No. DE-AC02-05CH11231,

\section{References}

1. Garrity G. NamesforLife. BrowserTool takes expertise out of the database and puts it right in the browser. Microbiol Today 2010; 7:1.

2. Benno Y, Watabe J, Mitsuoka T. Bacteroides pyogenes sp. nov., Bacteroides suis sp. nov., and Bacteroides helcogenes sp. nov., new species from abscesses and feces of pigs. Syst Appl Microbiol 1983; 4:396-407.

3. DeSantis TZ, Hugenholtz $P$, Larsen N, Rojas M, Brodie EL, Keller K, Huber T, Dalevi D, Hu P,
Lawrence Livermore National Laboratory under Contract No. DE-AC52-07NA27344, and Los Alamos National Laboratory under contract No. DE-AC0206NA25396, UT-Battelle and Oak Ridge National Laboratory under contract DE-AC05-000R22725, as well as German Research Foundation (DFG) INST 599/1-2.

Andersen GL. Greengenes, a Chimera-Checked $16 S$ rRNA Gene Database and Workbench Compatible with ARB. Appl Environ Microbiol 2006; 72:5069-5072. PubMed doi:10.1128/AEM.03006-05

4. Porter MF. An algorithm for suffix stripping. Program: electronic library and information systems 1980; 14:130-137.

5. Yarza P, Richter M, Peplies J, Euzeby J, Amann R, Schleifer KH, Ludwig W, Glöckner FO, Rosselló- 
Móra R. The all-species living tree project: A $16 \mathrm{~S}$ rRNA-based phylogenetic tree of all sequenced type strains. Syst App/ Microbiol 2008; 31:241250. PubMed doi:10.1016/j.syapm.2008.07.001

6. Lee C, Grasso C, Sharlow MF. Multiple sequence alignment using partial order graphs. Bioinformatics 2002; 18:452-464. PubMed doi:10.1093/bioinformatics/18.3.452

7. Castresana J. Selection of conserved blocks from multiple alignments for their use in phylogenetic analysis. Mol Biol Evol 2000; 17:540-552. PubMed

8. Stamatakis A, Hoover P, Rougemont J. A rapid bootstrap algorithm for the RAxML web-servers. Syst Biol 2008; 57:758-771. PubMed doi:10.1080/10635150802429642

9. Pattengale ND, Alipour M, Bininda-Emonds ORP, Moret BME, Stamatakis A. How Many Bootstrap Replicates Are Necessary? Lect Notes Comput Sci 2009; 5541:184-200. doi:10.1007/978-3-64202008-7_13

10. Liolios K, Chen IM, Mavromatis K, Tavernarakis N, Hugenholtz P, Markowitz VM, Kyrpides NC. The Genomes OnLine Database (GOLD) in 2009: status of genomic and metagenomic projects and their associated metadata. Nucleic Acids Res 2010; 38:D346-D354. PubMed doi:10.1093/nar/gkp848

11. Cerdeño-Tárraga AM, Patrick S, Crossman LC, Blakely G, Abratt V, Lennard N, Poxton I, Duerden B, Harris B, Quail MA, et al. Extensive DNA inversions in the $B$. fragilis genome control variable gene expression. Science 2005; 307:14631465. PubMed doi:10.1126/science. 1107008

12. Field D, Garrity G, Gray T, Morrison N, Selengut J, Sterk P, Tatusova T, Thomson N, Allen MJ, Angiuoli SV, et al. The minimum information about a genome sequence (MIGS) specification. Nat Biotechnol 2008; 26:541-547. PubMed doi:10.1038/nbt1360

13. Woese CR, Kandler O, Wheelis ML. Towards a natural system of organisms: proposal for the domains Archaea, Bacteria, and Eucarya. Proc Natl Acad Sci USA 1990; 87:4576-4579. PubMed doi:10.1073/pnas.87.12.4576

14. Garrity GM, Holt JG. The Road Map to the Manual. In: Garrity GM, Boone DR, Castenholz RW (eds), Bergey's Manual of Systematic Bacteriology, Second Edition, Volume 1. Springer, New York 2001:119-169.
15. Ludwig W, Euzeby J, Whitman WG. Draft taxonomic outline of the Bacteroidetes, Planctomycetes, Chlamydiae, Spirochaetes, Fibrobacteres, Fusobacteria, Acidobacteria, Verrucomicrobia, Dictyoglomi, and Gemmatimonadetes. http://www.bergeys.org/outlines/Bergeys_Vol_4 Outline.pdf. Taxonomic Outline 2008.

16. Garrity GM, Holt JG. Taxonomic Outline of the Archaea and Bacteria. In: Garrity GM, Boone DR, Castenholz RW (eds), Bergey's Manual of Systematic Bacteriology, Second Edition, Volume 1, Springer, New York, 2001, p. 155-166.

17. Skerman VBD, McGowan V, Sneath PHA. Approved Lists of Bacterial Names. Int J Syst Bacteriol 1980; 30:225-420. doi:10.1099/00207713$\underline{30-1-225}$

18. Pribram E. Klassification der Schizomyceten. Klassifikation der Schizomyceten (Bakterien), Franz Deuticke, Leipzig, 1933, p. 1-143.

19. Castellani A, Chalmers AJ. Genus Bacteroides Castellani and Chalmers, 1918. Manual of Tropical Medicine, Third Edition, Williams, Wood and Co., New York, 1919, p. 959-960.

20. Holdeman LV, Moore WEC. Genus I. Bacteroides Castellani and Chalmers 1919, 959. In: Buchanan RE, Gibbons NE (eds), Bergey's Manual of Determinative Bacteriology, Eighth Edition, The Williams and Wilkins Co., Baltimore, 1974, p. 385404.

21. Cato EP, Kelley RW, Moore WEC, Holdeman LV. Bacteroides zoogleoformans, Weinberg, Nativelle, and Prévot 1937) corrig. comb. nov.: emended description. Int J Syst Bacteriol 1982; 32:271-274. doi:10.1099/00207713-32-3-271

22. Shah HN, Collins MD. Proposal to restrict the genus Bacteroides (Castellani and Chalmers) to Bacteroides fragilis and closely related species. Int J Syst Bacteriol 1989; 39:85-87. doi:10.1099/00207713-39-1-85

23. Validation List no. 12. Validation of the publication of new names and new combinations previously effectively published outside the IJSB. Int J Syst Bacteriol 1983; 33:896-897. doi:10.1099/00207713-33-4-896

24. Classification of bacteria and archaea in risk groups. http://www.baua.de TRBA 466.

25. Ashburner M, Ball CA, Blake JA, Botstein D, Butler $\mathrm{H}$, Cherry JM, Davis AP, Dolinski K, Dwight SS, Eppig JT, et al. Gene Ontology: tool for the unification of biology. Nat Genet 2000; 25:25-29. $\underline{\text { PubMed doi:10.1038/75556 }}$ 
26. Saito $H$, Miura K. Preparation of transfroming deoxyribonucleic acid by phenol treatment. Biochim Biophys Acta 1963; 72:619-629. PubMed doi:10.1016/0926-6550(63)90386-4

27. Klenk HP, Goeker M. En route to a genome-based classification of Archaea and Bacteria? Syst Appl Microbiol 2010; 33:175-182. PubMed doi:10.1016/j.syapm.2010.03.003

28. Wu D, Hugenholtz P, Mavromatis K, Pukall R, Dalin E, Ivanova NN, Kunin V, Goodwin L, Wu $M$, Tindall BJ, et al. A phylogeny-driven genomic encyclopaedia of Bacteria and Archaea. Nature 2009; 462:1056-1060. PubMed doi:10.1038/nature08656

29. List of growth media used at DSMZ: http://www.dsmz.de/microorganisms/media list.p hp.

30. Gemeinholzer B, Dröge G, Zetzsche H, Haszprunar G, Klenk HP, Güntsch A, Berendsohn WG, Wägele JW. The DNA Bank Network: the start from a German initiative. Biopreservation and Biobanking. (In press).

31. DNA Bank Network. http://www.dnabanknetwork.org

32. DOE Joint Genome Institute. http://www.jgi.doe.gov

33. Phrap and Phred for Windows. MacOS, Linux, and Unix. http://www.phrap.com
34. Zerbino DR, Birney E. Velvet: algorithms for de novo short read assembly using de Bruijn graphs. Genome Res 2008; 18:821-829. PubMed doi:10.1101/gr.074492.107

35. Han C, Chain P. 2006. Finishing repeat regions automatically with Dupfinisher. in Proceeding of the 2006 international conference on bioinformatics \& computational biology. Edited by Hamid R. Arabnia \& Homayoun Valafar, CSREA Press. June 26-29, 2006: 141-146.

36. Lapidus A, LaButti K, Foster B, Lowry S, Trong S, Goltsman E. POLISHER: An effective tool for using ultra short reads in microbial genome assembly and finishing. AGBT, Marco Island, FL, 2008.

37. Hyatt $\mathrm{D}$, Chen GL, LoCascio PF, Land ML, Larimer FW, Hauser LJ. Prodigal: prokaryotic gene recognition and translation initiation site identification. BMC Bioinformatics 2010; 11:119. PubMed doi:10.1186/1471-2105-11-119

38. Pati A, Ivanova NN, Mikhailova N, Ovchinnikova G, Hooper SD, Lykidis A, Kyrpides NC. GenePRIMP: a gene prediction improvement pipeline for prokaryotic genomes. Nat Methods 2010; 7:455-457. PubMed doi:10.1038/nmeth.1457

39. Markowitz VM, Ivanova NN, Chen IMA, Chu K, Kyrpides NC. IMG ER: a system for microbial genome annotation expert review and curation. Bioinformatics 2009; 25:2271-2278. PubMed doi:10.1093/bioinformatics/btp393 